\title{
XLIV. On the specific heat of the gases
}

\section{W.T. Haycraft Esq.}

To cite this article: W.T. Haycraft Esq. (1824) XLIV. On the specific heat of the gases, Philosophical Magazine Series 1, 64:318, 262-269, DOI: 10.1080/14786442408644598

To link to this article: http://dx.doi.org/10.1080/14786442408644598

曲 Published online: 27 Jul 2009.

Submit your article to this journal $\widetilde{ }$

Џ Article views: 2

Q View related articles $₫$ 


\section{[ 262$]$ \\ XLIV. On the Specific Heat of the Gases. By W. T. HaY- CrAFT, Esq.}

[Concluded from p. 207.]

Experiments on Hydrogen.

HYDROGEN gas was procured from the decomposition of water by means of sulphuric acid and zinc. The part B Plate II., was filled with the same, and the following experiments were made. No. 1.

In this experiment the calorimeters were filled with water of the same temperature, and the process was conducted on rather a different principle than the former; namely, it was continued until the calorimeters ceased to rise in temperature, or, rather, till the temperature began to fall. This latter circumstance would take place when the heat communicated by the gas was exactly equal to that abstracted by the colder surrounding medium. The number of degrees of temperature, then, which each gas would sustain in its calorimeter, will be the ratio of its power for giving out heat, and consequently of its capacity for caloric.

The temperature of calorimeter $\mathrm{A}$, at the beginning of the experiment, was about $50^{\circ}$, and after 105 minutes the temperature of calorimeter $A$ was $82^{\circ} \frac{15}{2}$, and that of $\mathrm{B}$, containing hydrogen gas, was $82 \frac{1}{2} \frac{1}{2}$, and the surrounding medium $60^{\circ} \frac{9}{20}$, indicating the comparative capacity of hydrogen to be 98.64, being a difference so trifling, that it may be regarded as the same as that of atmospheric air; if we make allowance for the evident greater ratio in its heating, and the smaller ratio of its rate of cooling at the end of the experiment. This will be seen by the following table.

\begin{tabular}{|c|c|c|}
\hline \\
\hline & & 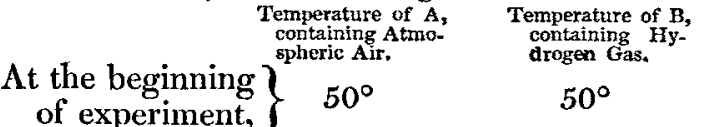 \\
\hline In 5 minutes, & 59 & $58 \cdot 6$ \\
\hline In 10 minutes, & $67 \frac{16}{2}$ & $66 \frac{14}{2}$ \\
\hline In 15 minutes, & $71 \frac{1}{2} \frac{6}{0}$ & $71 \frac{4}{20}$ \\
\hline In 20 minutes, & & $73 \frac{40}{20}$ \\
\hline In 25 minutes, & $77 \frac{16}{20}$ & $76^{2}$ \\
\hline In 30 minutes, & $79^{20}$ & $77 \frac{6}{20}$ \\
\hline In 35 minutes, & $80 \frac{1}{2} \frac{2}{x}$ & $78 \frac{10}{20}$ \\
\hline In 40 minutes, & $81 \frac{12}{2} \frac{2}{0}$ & $80 \frac{3}{20}$ \\
\hline In 45 minutes, & $82 \frac{8}{20}$ & $81^{2}$ \\
\hline In 50 minutes, & $83^{60}$ & $82 \frac{9}{29}$ \\
\hline In 55 minutes, & 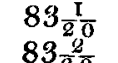 & $\begin{array}{l}82 \frac{8}{20} \\
8212\end{array}$ \\
\hline In 65 minutes, & $\begin{array}{l}80 \frac{20}{3} \\
830\end{array}$ & $\begin{array}{l}82 \frac{10}{2} \frac{0}{0} \\
82 \frac{10}{0}\end{array}$ \\
\hline In 70 minutes, & $82 \frac{16}{26}$ & $82 \frac{1}{2} \frac{0}{0}$ \\
\hline
\end{tabular}




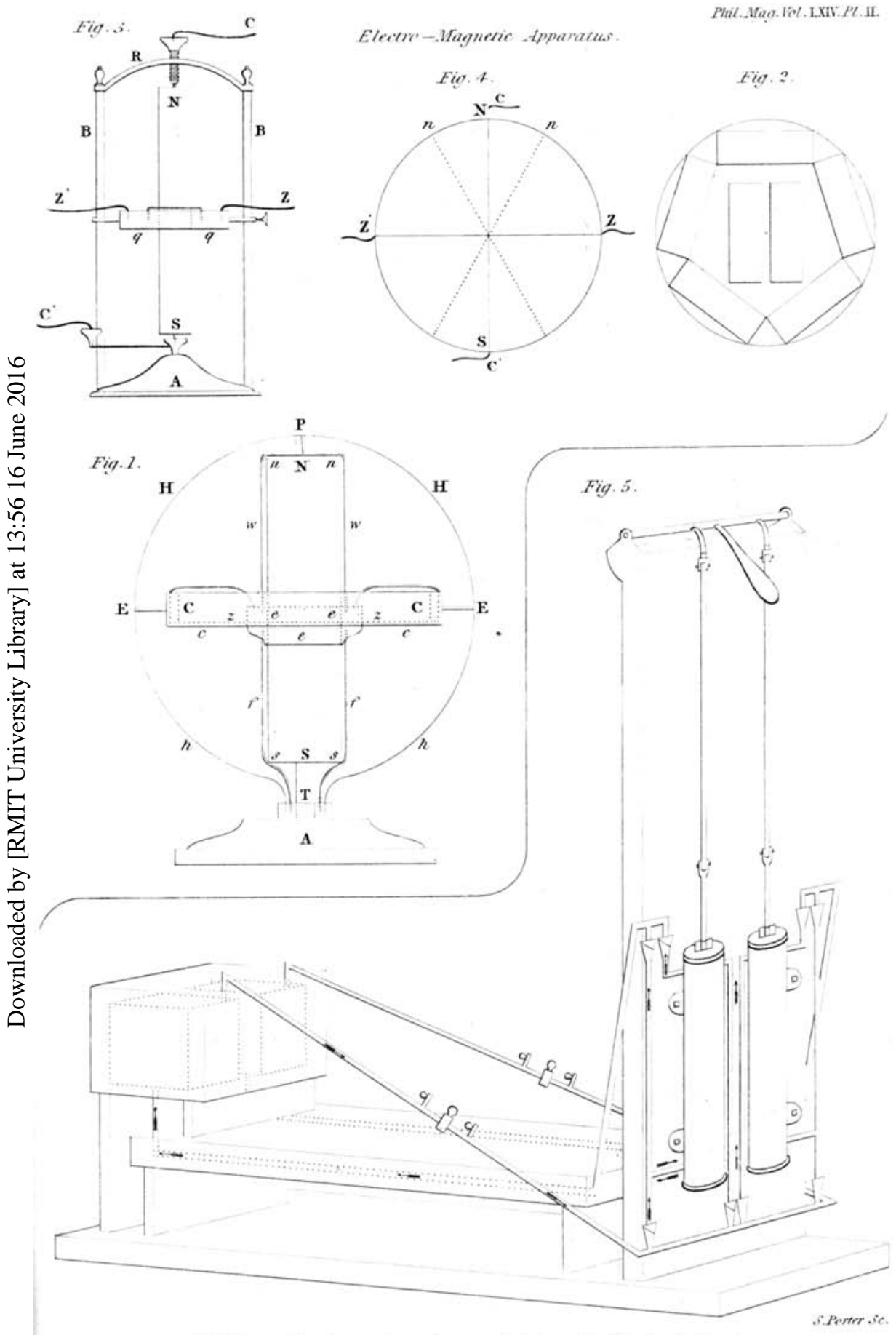

Wr Haycratts Apparatus tor ascertmining the Heat of Gases. 
At the beginning
of experiment,

Temperature of A,
containing Atmospheric Air.

After 5 minutes,

After 10 minutes,

After 15 minutes,

After 20 minutes,

After 25 minutes,

No. 2.

This last experiment was conducted as the former ones.

The air appeared, after the experiments, to contain 88 per cent. of hydrogen gas, as indicated by explosion with oxygen gas*.

In these two experiments it may be observed, that the watery vapour which may be presumed to be in the hydrogen gas, before it had been sufficiently exposed to the drying influence of the muriate of lime, seemed to decrease in specific heat, exactly contrary to what might be expected. In the first experiment, at the expiration of the first five minutes, it had a capacity of 9222 , pretty nearly the same as indicated in the experiments of Messrs. De la Roche and Berard; but in proportion as the experiment had advanced, and the hydrogen had been exposed longer to the muriate of lime, its specific heat approached to that of atmospheric air, till, at the end of the experiment, they were quite equal.

No. 2. was performed upon the same hydrogen, in its driest state; and throughout the whole experiment it indicated also a capacity equal to the standard. In this experiment I know of no source of fallacy, as the gases, while entering into the calorimeters, were of exactly the same temperature, and care was taken to ensure accuracy.

\section{Azote.}

\section{Of azote I shall merely state, that last year I performed}

- The apparatus which I found most convenient for exploding gases, is a modification of Dr. Ure's syphon eudiometer. It consists of a hole bored in the solid bottom of a mercurial trough, representing an inverted syphon; one end of which opens into the part containing mercury, and the other through the edge of the trough to the open air. To the latter opening is cemented an open glass tube; and to the former a common graduated eudiometer is made to fit accurately. When this apparatus is used, the graduated tube is filled in the usual way, and applied to the opening communicating with the trough. Mercury is poured into the other tube, to the same height as that contained in the graduated one. The finger is then applied to the open tube, and the electric spark passed. After the explosion, more mercury is poured into the open tube, to the same height that it had risen in the eudiometer, after which the degrees are read off. 
similar experiments upon this gas, the results of which were perfectly analogous with those now detailed; and as all the experiments agree that it has by volume the same specific heat as atmospheric air, namely 1000 , I thought it needless to repeat them.

\section{Carburetted Hydrogen.}

In my former experiments on carburetted hydrogen, procured from the decomposition of sea-coal, I concluded that it also had the same capacity as atmospheric air; but I have since found that the capacity of this gas varies extremely, according to the modes in which it is procured. That produced from sea-coal seems to have a capacity nearly equal to the standard; that from the decomposition by heat of animal fat, has a much greater capacity. From the following experiments, however, it will appear that olefiant gas owes its increased capacity to the empyreumatic or ethereal vapour with which it is usually combined.

No. 1.

This experiment $I$ conducted in the same way as No. 1. on hydrogen gas. The part $\mathrm{B}$ was filled with olefiant gas obtained from the gas-pipes of a public Company. The calorimeters at the beginning of the experiment contained water of the temperature of $50^{\circ}$. At the end of 50 minutes the calorimeter $A$ had acquired its utmost temperature of $92^{\circ}{ }^{7} \overline{0}$, and of B that of $93^{\circ} \frac{1}{2} \frac{2}{0}$; the surrounding medium being $66^{\circ} \frac{8}{20}$.

No. 2.

The calorimeters were of a temperature of $52^{\circ} \frac{5}{20}$ at the beginning of the experiment: after 55 minutes, the calorimeter A had acquired a temperature of $92^{\circ} \frac{1}{2} \frac{0}{0}$, and $B$ that of $94^{\circ} \frac{4}{2} 5$; the surrounding medium being $65^{\circ}$. The average result of these experiments, Nos. 1. and 2., indicates the specific heat of olefiant gas to be 10559. Though the results of these two experiments do not quite agree with those I formerly made, yet the difference is very trifling, and may be supposed to arise from the greater freedom of the gas I formerly made use of, from empyreumatic vapour. This will appear probable from the following experiments.

\section{No. 3.}

The part of the apparatus $B$ was filled with carburetted hydrogen, procured by the destructive distillation of muttonsuet. The calorimeters were filled with water of the temperature of $50^{\circ} \frac{1}{2} \frac{5}{0}$. At the end of 40 minutes, the calorimeter through which the olefiant gas passed had acquired its extreme temperature of $95^{\circ}$, the other that of $88^{\circ} \frac{10}{2} 0$; the surrounding medium being $65^{\circ} \frac{2}{20}$; indicating the specific heat of olefiant gas to be 12777 .

That 
That the gas procured from animal fat contains more empyreumatic vapour, is evident from its sensible qualities, which may account for its greater specific heat, compared with that procured from sea-coal. The gases, at the end of the experiment, were exactly of the same temperature as when entering into the calorimeters.

No. 4 .

The last experiment was repeated, except that the olefiant gas was procured from alcohol and sulphuric acid. After 25 minutes, the calorimeter $A$ had assumed the temperature of $74^{\circ} \frac{4}{20}$, and calorimeter $B$ that of $75^{\circ} \cdot 10$; the surrounding medium being $54^{\circ}$; indicating the capacity of olefiant gas to be 10643 .

No. 5.

The last experiment was repeated, and gave a result of 10674 ; the medium result of experiments Nos. 4 and 5, being 10658, indicating the capacity of olefiant gas procured from alcohol and from sea-coal to be almost exactly the same.

No. 6.

Wishing to ascertain if the ethereal or empyreumatic vapour in olefiant gas affected its specific heat, I poured a few drops of sulphuric ether into the part of the apparatus containing atmospheric air, that the latter, as well as the olefiant gas, being equally saturated with the vapours of ether, it might be ascertained what effect that condition might have on the capacities of the gases. The part B contained the olefiant gas as before. After 40 minutes, both the calorimeters had acquired a temperature of $85^{\circ} \frac{3}{20}$, the surrounding medium being $61^{\circ} \frac{4}{2} \frac{4}{0}$. The inference, then, may fairly be made, that it is the combined vapour that increases the specific heat of olefiant gas.

\section{Experiments on the Air of Respiration.}

Having last year made more than ten experiments which prove that the mixtures of carbonic acid with atmospheric air exposed freely to water, and at a temperature of about $100^{\circ}$, had a much less capacity for heat than atmospheric air had, under ordinary circumstances, and this curious fact seeming to throw some light upon the physiology of animal respiration, I filled the part B with air from the lungs, and the part A with atmospherical air.

The heating apparatus was kept, by means of a lamp, at the temperature of between $97^{\circ} \frac{10}{2} \frac{0}{6}$ and $100^{\circ} \frac{10}{2}$. After the end of 35 minutes, the calorimeter through which the air of respiration passed attained the temperature of $59^{\circ} \frac{4}{25}$, and the other that

Vol. 64. No. 318. Oct. 1824. L 1 of 
of $61^{\circ} \frac{4}{20}$; the surrounding medium being $54^{\circ} \cdot 16$, indicating the air of respiration to be 6875 .

No. 2.

The last experiment was repeated, when the calorimeter arose from $56^{\circ} \frac{2}{20}$ to $58^{\circ} \frac{1}{2} \frac{0}{0}$, and $\mathrm{B}$ from $56^{\circ} \frac{3}{20}$ to $57^{\circ} \frac{1}{2} \frac{6}{0}$, indicating the capacity of the air of respiration to be, as in the last experiment, 6875 .

It may not be improper in this place to state, that in my former experiments mixtures of carbonic acid and atmospheric air, under different conditions of temperature, and combination with watery vapour, had relative capacities of $3333,66666^{\circ} 9999$, and 13333. It was $m y$ intention to have repeated those experiments in such a way as to ascertain the precise conditions under which these changes of capacities took place; but, from various engagements; I am unable to do so. I may remark, however, that the last two experiments seem to indicate, that the air of respiration enters into the second of this series, making allowance for the difference of the standard of comparison; this being in my former experiments common undried atmospherical air, while the standard of the latter was the same air carefully dried.

There is also a curious coincidence between this last-mentioned series of capacities of gas in different states of combination with water, and the expansive forces of air combined also with different proportions of watery vapours. Having procured a glass globe, to which a small stem was connected, in such a way that mercury contained in the hollow ball would rise into the stem upon any increase of the expansive force of the air contained in the ball, I filled the latter with air at a temperature of $60^{\circ}$; after which the ball was immersed into boiling water. In a short time the mercury rose into the stem to the height of 7 inches. The experiment was repeated, excepting that a few drops of water were put, together with the air, into the ball. The mercury, after the immersion of the ball in boiling water, rose to 21 inches. Afterwards, on passing a quantity of water into the ball, the mercury, after its immersion, rose to 28 inches. Some months afterwards, on repeating the experiment, the mercury rose in one instance to 14 inches. Thus we have a series of expansive forces of air united to watery vapour of $7,14,21$ and 28 inches: it was upon this principle that I contrived an air thermometer. The form of it is similar to that of the differential thermometer invented by Professor Leslie. One ball contained atmospherical air dried by means of muriate of lime; the other contained air in its usual state. Interposed between the balls was a co- 
lumn of the volatile spirit of turpentine. Upon any rise of the temperature of the atmosphere, the column immediately rose at the side of the dry ball. After some time, however, the instrument seemed to have lost its power; and after a still longer period, the ball containing dry air had the greater expansive force. This I accounted for by supposing, that the vapour of turpentine had in process of time combined with the dry air, and had given it its greater expansive power. This thermometer is now a remarkably delicate one, though its degrees are of very unequal length, and appear to vary by lapse of time. Probably hydrogen gas contained in two platina balls, in one of which a little mercury might be placed, connected together in the same way, would make an accurate pyrometer, indicating temperatures as high as the melting point of platina.

There is another condition under which air is capable of a great variety of specific heats, namely, when it exists in different degrees of density, whether arising from pressure or other causes. The increased capacity of air, when under lesser degrees of atmospheric pressure, has been properly made use of to explain the extreme cold which exists in high regions; and its decreased capacity under mechanical pressure, also satisfactorily accounts for the heat evolved under that condition. This principle, so far as I know, has not been used to explain one cause of the intense heat produced during the combustion of gunpowder and other explosive mixtures. If we reflect a moment, however, we shall perceive that the resistance of the pressure of the atmosphere to the expansion of the nascent gases produced by the combustion, will cause them to exist in a state of greater density than when the resistance of the atmosphere has been finally overcome. It is during this state of potential compression, if I may use the term, that the intense heat is produced. After the first explosion, however, the gaseous products will expand, and then there will necessarily be an absorption of caloric, and consequently comparative coldness, produced. In order to ascertain whether there is a permanent evolution of caloric, occasioned by the combustion of gunpowder, I made the following experiment.

Having a receiver containing 528 cubic inches, filled with water of a temperature of $52^{\circ}$, placed in a pneumatic trough, the surrounding atmosphere being also $52^{\circ}$, I introduced 240 inches of the aêriform fluids, produced during the combustion of that composition of gunpowder which is used for pyrotechnical purposes. After the explosion, the gas in the upper part 
of the receiver had acquired a temperature of nearly $54^{\circ}$, and the water not so much. This experiment shows, that though heat is evolved in the combustion of gunpowder, its quantity is not nearly so great as has been imagined. Again, if we consider that the products of the combustion of gunpowder have not, by direct experiment, been proved to have a greater specific heat than the ingredients of that composition, the phænomenon of heat being produced during that combustion should not be urged as an objection to the hypothesis of Black and Crawford. Indeed it appears very probable, from the inspection of the table of specific heats of different bodies, that those elastic products have a less capacity than the ingredients of gunpowder, from which they are produced. For example, azote, which composes two-thirds of the elastic products, has a capacity of 2669 , and carbonic acid, comprising one-third of the products, if my experiments are to be trusted to, has a capacity of only 1751 , water being 10000 . Nitric acid of a specific gravity of J,1354 has a capacity of 5760 . The azote, therefore, and oxygen, which is produced from the decomposition of one of the ingredients forming the elastic products of not half the specific heat of that ingredient, should, according to the hypothesis of Black, evolve heat. This might take place even if we make allowance for the lesser capacity which nitric acid has in its state of one of the ingredients of the nitrate of potash.

The same condition of potential compression may also contribute to the intense heat which takes place in a blast-furnace. This heat is known by all conversant with the phænomenon to be, not in a ratio of the fuel consumed, but of some compound ratio. This may be explained in the following manner: 1st, $A$ quantity of air is forced into contact with the coals in a state of ignition, and its temperature is suddenly raised extremely high. $2 \mathrm{~d}$, In this condition, were it not for the pressure of the atmosphere, it would become as suddenly expanded. 3d, Had this expansion taken place, it would have acquired an increased capacity, and would consequently have absorbed a considerable portion of the caloric evolved by the combustion, tending thereby to lessen the capacity of the heat. 4th, But the heated air being prevented by the pressure of the atmosphere from expanding in a ratio equal to the temperature acquired, the absorption of caloric is lessened, and a greater proportion of the heat of combustion is rendered free. Thus, although the total quantity of caloric evolved at, and consequently to combustion, may be in a direct ratio of the quantity of fuel consumed; yet the intensity of the thermometrical heat at the moment, 
ment, and at the place of combustion, will be greater in a compound ratio, directly as the pressure of the atmosphere, and inversely as the times of expansion of the air employed in the blast. These times are, of course, inversely as the intensity of the blast. The thermometrical heat, then, at the moment and place of combustion, will be in a compound ratio of the quantity of fuel consumed, the weight of the atmosphere, and the quantity of air employed in the blast in a given time. The same rule will hold even in what are called Chimney Furnaces; and it is ascertained by experience, that those furnaces of steam-engines through which a greater quantity of air passes in a given time, consume a proportionally less quantity of fuel to produce the same effect. Probably blast-furnaces might be advantageously employed in lessening the quantity of fuel used for those valuable machines.

Although, according to the foregoing experiment, it appears contrary to my original expectation, that, by volume, oxygen gas has the same specific heat as carbonic acid, it by no means follows that caloric should not be evolved during the formation of the latter by combustion. This formation does not consist of a conversion of oxygen into carbonic acid, but of a union of two ingredients into a compound, having an absolute capacity for caloric equal to one of the ingredients only, namely, the oxygen gas; consequently the whole absolute heat of the carbon is rendered free.

The direct results of these experiments show, that the specific heats of all the gases experimented upon are to each other inversely as their specific gravities; and, 2dly, That different states of combinations of the gases with aqueous and other vapours, affect the capacities of the gases, and that probably, in some instances, in a regular arithmetical progression, corresponding with the arithmetical rate of expansive force of the gases in different states of combination with vapour. The most interesting result to the physiologist is, that the air of respiration, at a temperature of between $100 \frac{1}{2}{ }^{\circ}$ and $95^{\circ}$, has a less specific heat than atmospherical air. Many experiments were made which are not here detailed, which showed that the air of respiration, at the temperature of $102^{\circ}$ and upwards, and of $91^{\circ}$ and downwards, had a capacity the same as that of atmospherical air. I should feel a hesitation in stating these results, had not experiments, very often repeated, during a course of several months, warranted me in my conclusions. 